\title{
VERSIÓN ESPAÑOLA DEL CUESTIONARIO EPQR-ABREVIADO (EPQR-A) (II): REPLICACIÓN FACTORIAL, FIABILIDAD Y VALIDEZ
}

\author{
BONIFACIO SANDIN, ROSA M. VALIENTE, MARGARITA OLMEDO, \\ PALOMA CHOROT y MIGUEL A. SANTED \\ Universidad Nacional de Educación a Distancia
}

(Aceptado en agosto de 2002)

\begin{abstract}
En el presente estudio se proporciona evidencia empírica relacionada con la validación de la versión española del Eysenck Personality Questionnaire Revised-Abbreviated (EPQR-A). Una muestra constituida por 199 estudiantes universitarios (rango de edad entre 18 y 39 años) cumplimentó los siguientes cuestionarios (versión española): EPQR-A, Eysenck Personality Questionnaire (EPQ), State-Trait Anxiety Inventory (STAI), Positive and Negative Affect Schedule (PANAS), Anxiety Sensitivity Index (ASI) y Penn State Worry Questionnaire (PSWQ). Utilizando análisis factorial, los autores replicaron la estructura del EPQR-A previamente obtenida por Sandin et al. [Sandin, B., Valiente, R.M., Chorot, P., Olmedo, M., y Santed, M.A. (2002). Versión española del cuestionario EPQR-Abreviado (EPQR-A) (I): Análisis exploratorio de la estructura factorial. Revista de Psicopatología y Psicología Clínica, 7, 195-205], validando la unidimensionalidad de las cuatro subescalas del EPQR-A (extraversión, neuroticismo, psicoticismo y sinceridad). Así mismo, los resultados proporcionan apoyo emṕrico a favor de la fiabilidad y validez de la versión española del EPQR-A .
\end{abstract}

Palabras clave: EPQR-A, EPQ, extraversión, neuroticismo, psicoticismo, análisis factorial, ASI, PSWQ, PANAS, STAI.

\section{Spanish version of the Eysenck Personality Questionnaire-Revised (EPQR-A) (II):} Factor analysis replication, reliability and validity

In the present study we report empirical evidence concerning validation of the Spanish Eysenck Personality Questionnaire Revised-Abbreviated (EPQR-A). A sample of 199 university students (ranging in age from 18 to 39 years) completed the Spanish versions of the EPQR-A, the Eysenck Personality Questionnaire (EPQ), the State-Trait Anxiety Inventory (STAI), the Positive and Negative Affect Schedule (PANAS), the Anxiety Sensitivity Index (ASI) and the Penn State Worry Questionnaire (PSWQ). Using factor analysis, the authors replicated the structure of the EPQR-A previously reported by Sandin et al. [Sandin, B., Valiente, R.M., Chorot, P., Olmedo, M., y Santed, M.A. (2002). Versión española del cuestionario EPQR-Abreviado (EPQR-A) (I): Análisis exploratorio de la estructura factorial. Revista de Psicopatología y Psicologia Clinica, 7, 195-205] providing evidence for the unidimensionality of the four EPQR-A subscales (extraversion, neuroticism, psychoticism and the lie scale). Results provide also empirical support for reliability and validity of the Spanish EPQR-A.

Key words: EPQR-A, EPQ, extraversion, neuroticism, psychoticism, factor analysis, ASI, PSWQ, PANAS, STAI.

\section{INTRODUCCIÓN}

Francis, Brown y Philipchalk (1992) desarrollaron el cuestionario de personalidad

Correspondencia: Bonifacio Sandín, Facultad de Psicología, Universidad Nacional de Educación a Distancia (UNED), Ciudad Universitaria $s / n, 28040$ Madrid.
Eysenck Personality Questionnaire Revised-Abbreviated (EPQR-A). Esta es una forma abreviada del EPQR, contiene sólo 24 items y permite evaluar cuatro subescalas, de las cuales tres se refieren a dimensiones de la personalidad (extraversión, neuroticismo y psicoticismo) y la última evalúa la tendencia a mentir o, más 
bien, a emitir respuestas de deseabilidad social (escala de sinceridad). El interés de este cuestionario es notable, sobre todo si tenemos en cuenta que otras formas del EPQ (EPQ, EPQR, EPQR-S) poseen un número de items considerablemente mayor, lo que dificulta su aplicación en muchos contextos de investigación o clínicos. (Para una información más extensa véase Sandín, Valiente, Chorot, Olmedo y Santed, 2002).

Datos sobre la versión española del EPQR-A fueron obtenidos por nuestro grupo recientemente (Valiente, Sandín, Chorot y Santed, 1996). En dicho trabajo presentamos información preliminar sobre la estructura factorial del cuestionario, así como sobre la fiabilidad y la validez. Los datos apoyaban claramente las dimensiones de neuroticismo y extraversión, y en menor grado las de sinceridad y psicoticismo, especialmente esta última.

En nuestro estudio, dirigido específicamente a examinar la estructura factorial de la versión española del EPQR-A (Sandín et al., 2002), comprobamos mediante análisis factorial exploratorio las dimensiones del cuestionario. Constatamos que (1) las dimensiones de extraversión y neuroticismo aparecían muy claramente definidas, (2) la dimensión de sinceridad aparecía bien definida, aunque en menor grado que las dos anteriores, y (3) la dimensión de psicoticismo presentaba una configuración factorial pobremente definida (los datos sólo apoyaban parcialmente la consistencia de la escala de psicoticismo). En suma, aunque el EPQR-A presentaba en general validez factorial, ésta era dudosa para la dimensión de psicoticismo. Tras haber sustituido dos items de esta subescala del cuestionario original por dos items del EPQ, también pertenecientes a la subescala de psicoticismo, obtuvimos una estructura factorial mucho más consistente con las cuatro subescalas o dimensiones que sub- yacen al EPQR-A; la mejora se produjo fundamentalmente en la dimensión de psicoticismo, aunque también mejoró la configuración del factor de sinceridad.

A partir de nuestros primeros trabajos con el EPQR-A (Valiente et al., 1996; Sandín et al., 2002) se concluye que la subescala de psicoticismo, tal y como aparece en su forma original en este cuestionario, es problemática psicométricamente $y$ debería utilizarse con cautela. No obstante, basándonos en nuestros datos más recientes (Sandín et al., 2002), la forma modificada de esta subescala posee una validez factorial aceptable y podría representar más fielmente la dimensión general de psicoticismo que subyace al EPQ o EPQR. En dicho estudio sugeríamos la conveniencia de utilizar esta forma modificada del EPQR-A, al menos si deseábamos incluir la variable de psicoticismo en el protocolo de evaluación.

Puesto que, aparte de nuestros trabajos, todos los estudios que conocemos sobre el EPQR-A han documentado bajos niveles de consistencia interna, fiabilidad y validez para la dimensión de psicoticismo (Francis et al., 1992; Katz y Francis, 2000; Caruso, Witkiewitz, Belcourt y Gottlieb, 2002; Shevlin, Bailey y Adamson, 2002), hemos centrado la presente investigación en la forma modificada del EPQR-A (Sandín et al., 2002). Basándonos, por tanto, en esta forma del EPQR$A^{1}$, mediante el presente estudio pretendemos: (1) obtener datos normativos (medias y desviaciones típicas), (2) replicar la estructura factorial obtenida inicialmente por Sandín et al. (2002), (3) obtener información sobre la fiabilidad de las subescalas (consistencia interna) y (4) examinar la validez convergente y divergente.

\footnotetext{
' A no ser que se especifique otra cosa, siempre que en este artículo hablemos de la versión española del EPQR-A nos referiremos a la forma modificada de dicha versión (véase el Anexo II en Sandín et al., 2002).
} 


\section{MÉTODO}

\section{Participantes}

La muestra estuvo compuesta por 199 estudiantes universitarios de la Universidad Autónoma de Madrid (UAM). La muestra incluía 149 mujeres $(74,8 \%)$ y 50 varones $(25,2 \%)$. Las medias de edad eran similares entre ambos grupos; la media de edad de las mujeres era de 21,5 $(D T=6,3)$; la media de edad del grupo de hombres era de $22,1(D T=7,1)$. El rango de edad para la muestra total osciló entre 18 y 39 años.

\section{Pruebas psicológicas}

Los participantes contestaron de forma colectiva en aulas docentes de la UAM a los cuestionarios siguientes:

Cuestionario EPQR-A [Eysenck Personality Questionnaire Revised-Abbreviated] (Francis et al., 1992). Se aplicó la versión española del cuestionario (Sandín, Valiente y Chorot, 1999a), la cual, como la versión original, consta de 24 items y cuatro subescalas (Extraversión, Neuroticismo, Psicoticismo y Sinceridad), correspondiendo 6 items a cada una de las subescalas. El formato de respuesta es de Sí (1) vs. No (0), con un rango de puntuaciones para cada subescala entre 0 y 6 . Las tres primeras subescalas indicadas miden rasgos de personalidad, mientras que la última evalúa la tendencia a mentir (en realidad evalúa la tendencia a emitir respuestas de deseabilidad social; respecto a esta subescala, aunque en la versión original se evalúa el nivel de mentiras, en la versión española, al igual que ocurre con el cuestionario $\mathrm{EPQ}$, se evalúa inversamente, esto es, se calcula el nivel de sinceridad). (La versión española del EPQR-A aplicada se incluye en el Anexo II de Sandín et al.
(2002), y consistió en la forma modificada del cuestionario].

Cuestionario EPQ [Eysenck Personality Questionnaire]. Se aplicó la versión española del cuestionario adaptada por TEA (Eysenck y Eysenck, 1983). Consta de 94 items y mide las cuatro mismas dimensiones que el EPQR-A. El formato de respuesta es similar a este último cuestionario (Sí/No).

Cuestionario de Ansiedad Estado-Rasgo STAI [State-Trait Anxiety Inventory]. Se utilizó la versión española adaptada por TEA (Spielberger, Gorsuch y Lushene, 1982). Aplicamos la Parte 2 del STAI, la cual evalúa específicamente el rasgo de ansiedad. Esta parte consta de 20 items, los cuales fueron contestados por los participantes indicando cómo se sentían habitualmente según una escala de 4 niveles que variaba entre 1 ("Casi nunca») y 4 ("Casi siempre»).

Escalas de Afecto Positivo y Negativo PANAS [Positive and Negative Affect Schedule] (Watson, Clark y Tellegen, 1988). Se aplicó la versión española, la cual ha sido validada por nuestro grupo (Joiner, Sandín, Chorot, Lostao y Marquina, 1997; Sandín, Chorot, Lostao, Joiner, Santed y Valiente, 1999). Consta de 20 items, 10 referidos al afecto positivo y 10 al afecto negativo. Cada ítem se puntúa según una escala de 1 ( «Nada o casi nada») a 5 («Muchísimo»).

Índice de Sensibilidad a la Ansiedad [ASI, Anxiety Sensitivity Index] (Peterson y Reiss, 1992). Aplicamos la versión española adaptada por nuestro grupo (Sandín, Valiente y Chorot, 1999b). La ASI contiene 16 items; cada ítem se puntúa según una escala que varía entre 0 («Muy poco») y 4 («Muchísimo»). Las propiedades psicométricas (fiabilidad, consistencia interna y validez) de la versión española han sido suficientemente 
documentadas (Sandín, Chorot y McNally, 1996, 2001).

\section{Cuestionario de Preocupación PSWQ} [Penn State Worry Questionnaire] (Meyer, Miller, Metzger, y Borkovec, 1990). Utilizamos la versión española de Sandín y Chorot (1991). Es una prueba de 16 items diseñado para evaluar el nivel general de preocupación patológica. Cada sujeto estima para cada ítem el nivel de preocupación según un rango de 1 («Nada típico en mí) y 5 ( «Muy típico en mí»). Datos sobre la fiabilidad y validez del cuestionario han sido presentados por Sandín et al. (1995).

\section{Tratamiento estadístico}

Para examinar la estructura factorial del EPQR-A se aplicaron análisis de componentes principales siguiendo los mismos criterios de extracción que hemos descrito previamente para nuestro anterior estudio (véase Sandín et al., 2002). Con objeto de comparar la estructura factorial del presente estudio con nuestros datos previos se calcularon los coeficientes de congruencia $\left(r_{c}\right)$. Para dar cuenta de los restantes objetivos del estudio calculamos las medias y desviaciones típicas (DT), los coeficientes alfa de Cronbach y los coeficientes de correlación de Pearson producto-momento.

Tabla 1. Análisis de componentes principales: Saturaciones obtenidas para los items del EPQR-A en cada uno de los cuatro factores después de la rotación ortogonal (varimax) $(N=199)$

\begin{tabular}{|c|c|c|c|c|c|}
\hline Item del EPQR-A & & Extraversión & Neuroticismo & Psicoticismo & Sinceridad \\
\hline 1. ¿Tiene cambios en su estado de ánimo? & $(\mathrm{N})$ & 0,02 & $\mathbf{0 , 5 3}$ & 0,22 & 0,15 \\
\hline 2. ¿Es una persona habladora? & $(E)$ & 0,72 & 0,01 & $-0,19$ & 0,01 \\
\hline 3. ¿Le molesta ver sufrir a alguien? & (P) & $-0,18$ & $-0,09$ & $\mathbf{0 , 5 8}$ & $-0,06$ \\
\hline 4. ¿Es una persona animada? & $(E)$ & $\mathbf{0 , 6 4}$ & $-0,13$ & 0,03 & 0,04 \\
\hline 5. ¿Ha deseado ayudarse a sí mismo? & $(\mathrm{S})$ & $-0,10$ & 0,08 & 0,08 & $\mathbf{0 , 5 1}$ \\
\hline 6. ¿Tomaría drogas? & (P) & 0,14 & $-0,11$ & $\mathbf{0 , 3 8}$ & 0,25 \\
\hline 7. ¿Ha acusado a otras personas? & $(S)$ & 0,07 & 0,13 & 0,17 & $\mathbf{0 , 5 6}$ \\
\hline 8. ¿Prefiere actuar a su modo? & (P) & 0,12 & 0,22 & $\mathbf{0 , 5 1}$ & 0,20 \\
\hline 9. ¿Se siente a menudo harto? & $(\mathrm{N})$ & $-0,13$ & $\mathbf{0 , 6 5}$ & $-0,16$ & 0,23 \\
\hline 10. ¿Ha cogido algo de otra persona? & $(\mathrm{S})$ & 0,02 & 0,12 & $-0,26$ & 0,48 \\
\hline 11. ¿Se considera nervioso/a? & $(\mathrm{N})$ & 0,12 & 0,64 & 0,13 & $-0,12$ \\
\hline 12. ¿El matrimonio es algo pasado de moda? & (P) & 0,11 & $-0,05$ & 0,47 & 0,19 \\
\hline 13. ¿Podría animar una fiesta? & (E) & 0,72 & $-0,05$ & 0,18 & $-0,07$ \\
\hline 14. ¿Es una persona preocupada? & $(\mathrm{N})$ & $-0,20$ & $\mathbf{0 , 5 4}$ & $-0,23$ & $-0,02$ \\
\hline 15. ¿Tiende a mantenerse en el anonimato? & (E) & 0,44 & 0,01 & 0,13 & 0,12 \\
\hline 16. ¿Se dedica demasiado a asegurar el futuro? & $(\mathrm{P})$ & $-0,06$ & 0,06 & $\mathbf{0 , 3 1}$ & $-0,02$ \\
\hline 17. ¿Ha hecho trampas jugando? & (S) & 0,18 & $-0,03$ & 0,03 & $\mathbf{0 , 5 8}$ \\
\hline 18. ¿Sufre de los nervios? & $(\mathrm{N})$ & 0,01 & $\mathbf{0 , 6 2}$ & 0,17 & 0,05 \\
\hline 19. ¿Se ha aprovechado de alguien? & $(\mathrm{S})$ & 0,13 & $-0,09$ & 0,27 & $\mathbf{0 , 5 4}$ \\
\hline 20. ¿Es una persona callada? & (E) & $\mathbf{0 , 7 5}$ & $-0,13$ & 0,03 & $-0,01$ \\
\hline 21. ¿Suele sentirse solo/a? & $(\mathrm{N})$ & $-0,21$ & $\mathbf{0 , 5 9}$ & $-0,09$ & 0,03 \\
\hline 22. ¿Es mejor seguir las normas sociales? & (P) & $-0,12$ & 0,11 & $\mathbf{0 , 5 8}$ & 0,12 \\
\hline 23. ¿Le consideran muy animado/a? & (E) & $\mathbf{0 , 7 7}$ & 0,01 & $-0,09$ & 0,02 \\
\hline 24. ¿Practica siempre lo que predica? & $(\mathrm{S})$ & $-0,12$ & 0,04 & 0,14 & $\mathbf{0 , 4 3}$ \\
\hline
\end{tabular}

Nota: Los items se indican de forma resumida (véase Sandín et al., 2002, Anexo II). Entre paréntesis se indica la subescala a la que corresponde cada ítem según el cuestionario original: $N=$ Neuroticismo, $E=E x t r a v e r s i o ́ n, P=$ Psicoticismo, $S=$ Sinceridad. En negrita se indican las saturaciones $\geq 0,30$. 


\section{RESULTADOS}

\section{Replicación factorial de la estructura $\operatorname{del} E P Q R-A$}

Con objeto de contrastar la estructura factorial obtenida en nuestro anterior estudio sobre el EPQR-A (Sandín et al., 2002, Tabla 2), a partir de las puntuaciones de los 24 items del cuestionario (versión modificada) aplicamos análisis de componentes principales y rotación varimax. Obtuvimos 4 factores que explicaban el $40,2 \%$ de la varianza total. Los resultados del análisis, después de haberse efectuado la rotación ortogonal, son los que indicamos en la Tabla 1.

Los datos que presentamos en dicha tabla sugieren una estructura factorial muy similar a la obtenida previamente por nosotros (Sandín et al., 2002) y es consistente con el modelo de cuatro dimensiones que subyace al EPQR-A. Tal y como ocurría en nuestro estudio previo, las dimensiones de extraversión y neuroticismo tienden a ser más consistentes que las de psicoticismo y sinceridad (en los factores de extraversión y neuroticismo los pesos factoriales relevantes suelen ser mayores y los irrelevantes menores). No obstante, en general se evidencia una estructura clara y consistente con los resultados obtenidos por Sandín et al. (2002, Tabla 2).

Para examinar empíricamente la similitud o invarianza entre ambas estructuras factoriales (i.e., la obtenida en el pre- sente estudio y la obtenida por Sandín et al., 2002) se calcularon los coeficientes de congruencia (Tucker, 1951; Wrigley y Neuhaus, 1955). Mediante esta prueba estadística podemos conocer hasta qué punto existe convergencia entre los factores obtenidos en ambos estudios. Los resultados de los análisis de congruencia están indicados en la Tabla 2. Puede observarse que los coeficientes de congruencia son especialmente elevados entre los factores equivalentes; salvo para los factores de psicoticismo, para los restantes factores los coeficientes de congruencia superan el valor de 0,90. En contraste, entre los factores no equivalentes los valores de $r_{c}$ suelen ser muy bajos; como cabría esperar, son más bajos cuando están implicados los factores de extraversión o neuroticismo. Estos resultados constituyen un claro apoyo a la similitud factorial entre ambas estructuras factoriales, lo cual es consistente con la hipótesis de convergencia de la estructura del EPQR-A a través de muestras independientes.

\section{Medias y desviaciones típicas}

En la Tabla 3 se indican las medias y desviaciones típicas correspondientes a las cuatro subescalas del EPQR-A y según grupos de sexo. En general puede observarse que las puntuaciones son muy similares entre los grupos de hombres y mujeres.

Tabla 2. Coeficientes de congruencia $\left(r_{c}\right)$ entre los factores obtenidos en el presente estudio y los obtenidos por Sandín et al. (2002, Tabla 2)

\begin{tabular}{lcccc}
\hline & \multicolumn{4}{c}{ Sandin et al. (2002) } \\
\cline { 2 - 5 } Presente estudio & Extraversión & Neuroticismo & Psicoticismo & Sinceridad \\
\hline Extraversión & $\mathbf{0 , 9 6}$ & 0,02 & 0,02 & 0,06 \\
Neuroticismo & $-0,05$ & 0,93 & 0,13 & 0,18 \\
Psicoticismo & 0,04 & $-0,01$ & 0,85 & 0,28 \\
Sinceridad & 0,04 & 0,17 & 0,29 & 0,92 \\
\hline
\end{tabular}

Nota: En negrita se indican los valores de $r_{\mathrm{c}}$ sobresalientes $(\geq 0,80)$. 
212 Bonifacio Sandin, Rosa M. Valiente, Margarita Olmedo, Paloma Chorot y Miguel A. Santed

Tabla 3. Medias y desviaciones típicas $(D T)$ para las variables del EPQR-A según sexo

\begin{tabular}{lccccc}
\hline & \multicolumn{3}{c}{$\begin{array}{c}\text { Varones } \\
(n=50)\end{array}$} & \multicolumn{3}{c}{$\begin{array}{c}\text { Mujeres } \\
(n=149)\end{array}$} & \\
\cline { 2 - 5 } Subescala & Media & $(D T)$ & Media & $(D T)$ & $t$ \\
\hline Extraversión & 2,70 & $(1,56)$ & 3,17 & $(1,69)$ & 1,53 \\
Neuroticismo & 3,70 & $(1,78)$ & 3,77 & $(1,89)$ & 0,11 \\
Psicoticismo & 1,71 & $(1,31)$ & 1,55 & $(1,16)$ & 0,16 \\
Sinceridad & 3,75 & $(1,62)$ & 3,01 & $(1,57)$ & $2^{*}$ \\
\hline
\end{tabular}

${ }^{*} p<0,05$.

Fiabilidad (consistencia interna) del EPQR-A

Calculamos la fiabilidad del EPQR-A a partir de los coeficientes alfa de Cronbach. Como se indica en la Tabla 4, los coeficientes son más elevados para las subescalas de neuroticismo y extraversión que para las subescalas de psicoticismo y sinceridad. Con objeto de contrastar la fiabilidad del EPQR-A y del $E P Q$, se indican también los coeficientes alfa de este último cuestionario, los cuales, como cabría suponer (por el mayor número de items), son superiores en todos los casos.

\section{Validez convergente y divergente del EPQR-A}

La validez convergente y divergente se obtuvo calculando los coeficientes de correlación de Pearson entre las subescalas del EPQR-A y otras variables relevantes (subescalas del EPQ, subescalas del PANAS, rasgo de ansiedad, sensibilidad a la ansiedad y preocupación) (véase la Tabla 5). Los coeficientes de correlación sugieren que la validez convergente y divergente del EPQR-A es aceptable, especialmente para las subescalas de extraversión y neuroticismo. Aparte de las correlaciones que se dan entre los dos cuestionarios de personalidad emparentados (EPQR-A y EPQ), merece la pena destacar las correlaciones entre las subescalas de neuroticismo y extraversión del EPQR-A y las restantes variables (afecto positivo y negativo, rasgo de ansiedad, etc.), lo cual corrobora la validez del cuestionario que nos ocupa.

\section{DISCUSIÓN}

Los datos obtenidos en el presente estudio se relacionan con los cuatro aspectos siguientes del EPQR-A (forma modificada; véase Anexo II de Sandín et al., 2002): (1) datos normativos, (2) replicación de la estructura factorial, (3) fiabilidad (consistencia interna) y (4) validez convergente y divergente.

Respecto al primer punto, se evidencia que las medias y desviaciones típicas son similares para los grupos de mujeres y hombres (sólo se obtienen diferencias significativas en la subescala de sinceridad, siendo mayor la puntuación en el grupo

Tabla 4. Coeficientes alfa de Cronbach correspondientes a las subescalas de los cuestionarios EPQR-A y EPQ

\begin{tabular}{lcc}
\hline & $E P Q R-A$ & $E P Q$ \\
\hline Extraversión & 0,74 & 0,85 \\
Neuroticismo & 0,78 & 0,86 \\
Psicoticismo & 0,63 & 0,74 \\
Sinceridad & 0,54 & 0,70 \\
\hline
\end{tabular}


Tabla 5. Coeficientes de correlación entre el EPQR-A y otras variables relevantes

\begin{tabular}{lcccc}
\hline & \multicolumn{4}{c}{ EPQR-A } \\
\cline { 2 - 5 } & Extraversión & Neuroticismo & Psicoticismo & Sinceridad \\
\hline EPQ: & & & & \\
Extraversión & $0,86^{* \star}$ & 0,04 & $-0,05$ & 0,03 \\
Neuroticismo & $-0,11$ & $0,79^{* *}$ & $-0,14$ & 0,08 \\
Psicoticismo & $-0,15$ & 0,10 & $0,54^{* *}$ & 0,21 \\
Sinceridad & 0,04 & 0,06 & 0,20 & $0,66^{\star *}$ \\
PANAS: & & & & \\
Afecto negativo & $-0,06$ & $0,68^{\star *}$ & $-0,06$ & $-0,02$ \\
Afecto positivo & $0,57^{* *}$ & $-0,12$ & $-0,09$ & $-0,05$ \\
Ansiedad (STAI) & $-0,25^{*}$ & $0,69^{\star *}$ & 0,09 & 0,10 \\
Sensibilidad a la ansiedad (ASI) & 0,11 & $0,30^{*}$ & 0,16 & 0,09 \\
Preocupación (PSWQ) & 0,07 & $0,66^{* *}$ & 0,08 & 0,04 \\
\hline
\end{tabular}

" $p<0,01,{ }^{\star *} p<0,001$

de varones). En realidad nos sorprende que los principales estudios sobre el EPQR-A no hayan proporcionado información sobre las medias y desviaciones típicas (Francis et al., 1992; Forrest et al., 2000; Shevlin et al., 2002), lo cual dificulta poder contrastar nuestros resultados. Maltby y Talley (1998) indicaron las medias y desviaciones típicas, si bien para el EPQR-A junior, a partir de una muestra de chicos entre 12 y 15 años de edad. Estos autores obtuvieron puntuaciones medias significativamente más elevadas en las chicas para la variable de neuroticismo y en los chicos para la variable de psicoticismo. Aunque nuestros datos basados en el EPQR-A no sugieren marcadas diferencias entre varones y mujeres, a veces se ha sugerido que, partiendo fundamentalmente de evidencia basada en el EPQ, las mujeres suelen puntuar de forma más elevada en neuroticismo y mentiras y los hombres en psicoticismo (Francis, 1993; Forrest et al., 2000). Sin embargo, como han sugerido Forrest et al. (2000) para el EPQR-A, la magnitud real de estas diferencias es relativamente pequeña y trivial (p. 586).

En relación con el segundo aspecto, nuestros resultados proporcionan evidencia a favor de la validez de la estructura factorial del EPQR-A, tal y como queda de manifiesto a través de nuestro análisis factorial y de los análisis de congruencia. Una vez más se pone de relieve la mayor consistencia de las dimensiones de extraversión y neuroticismo, tal y como vimos en nuestro estudio inicial sobre la estructura factorial del cuestionario; los datos corroboran nuestros resultados previos obtenidos a partir de la forma modificada del EPQR-A (Sandín et al., 2002). Los análisis de congruencia resultan particularmente ilustrativos de la replicación factorial y discriminación entre los factores, destacando de forma especial la evidencia concerniente a las subescalas de extraversión y neuroticismo.

En lo que concierne al punto tercero, nuestros datos sobre los coeficientes de fiabilidad sugieren niveles entre buenos y aceptables para las subescalas de extraversión y neuroticismo, y ligeramente bajos para las subescalas de psicoticismo y sinceridad. No obstante, considerando el bajo número de items (6) en cada subescala, los niveles de fiabilidad obtenidos para estas dos últimas subescalas podrían considerarse como admisibles. Los datos sobre fiabilidad, por otra parte, concuerdan con los referidos por Francis et al. (1992), si bien estos autores obtuvieron niveles más bajos que nosotros para la subescala de psicoticismo (los 
niveles de estos autores oscilaron entre 0,33 y 0,52 ), lo cual podría deberse a los cambios que hemos efectuado en dicha subescala. Finalmente, cabe comentar que resulta coherente que los valores correspondientes al EPQ sean superiores a los del EPQR-A, dado el mayor número de items del primer cuestionario.

Como última cuestión relacionada con el presente estudio, pretendíamos examinar la validez convergente y divergente del EPQR-A. En primer lugar observamos elevadas correlaciones entre las subescalas del EPQR-A y las subescalas homólogas del EPQ, y bajas o despreciables correlaciones entre las subescalas no homólogas. Este fenómeno se produce de forma más marcada para las subescalas de extraversión y neuroticismo. En lo que concierne a estas dos subescalas, podría afirmarse que, dadas las correlaciones obtenidas, el EPQR-A es una prueba muy similar al EPQ. En contraste, las subescalas del EPQR-A relativas al psicoticismo y la sinceridad no parecen representar tan bien a sus análogas del EPQ, si bien las correlaciones entre ambas son elevadas y sugieren que miden constructos en gran medida equivalentes. Estos resultados son consistentes con los datos publicados por Francis et al. (1992), quienes obtuvieron unas correlaciones entre ambas formas del cuestionario que oscilaron entre 0,84 y 0,90 , excepto para la subescala de psicoticismo que oscilaron entre 0,44 y 0,52 (valores estos ligeramente inferiores a la correlación obtenida en el presente estudio).

Aparte de examinar las correlaciones entre ambas formas del cuestionario (i.e., EPQR-A y EPQ), con objeto de obtener información adicional sobre la validez del EPQR-A se calcularon las correlaciones de esta prueba con otras pruebas psicológicas relevantes. Las correlaciones entre las subescalas del EPQR-A y otras variables emocionales (afecto positivo, afecto negativo, rasgo de ansiedad, sensi- bilidad a la ansiedad y preocupación) sugieren que el EPQR-A posee aceptables niveles de validez convergente y divergente, ya que: (a) el neuroticismo correlaciona de forma elevada con afecto negativo, rasgo de ansiedad y preocupación ansiosa, (b) la extraversión correlaciona de forma elevada con afecto positivo, y (c) las restantes correlaciones son bajas o despreciables. La baja, aunque significativa, correlación entre neuroticismo y sensibilidad a la ansiedad es congruente considerando la naturaleza de este último constructo (Sandín et al., 1996, 2001).

Como conclusión general, el presente estudio, que es una continuación de nuestro anterior trabajo (Sandín et al., 2002), aporta evidencia empírica a favor de la versión española del EPQR-A, evidencia que concierne a la estructura factorial, fiabilidad y validez, además de informar sobre medias y desviaciones típicas de las subescalas. Partiendo de los datos que hemos presentado en este y en nuestro anterior estudio podríamos decir que la forma EPQR-A refleja bastante bien las características de su antecesor el EPQ (y por generalización el EPQR), tanto en lo que concierne a las dimensiones (estructura factorial) o consistencia de las subescalas como en lo relativo a la fiabilidad y validez. Esta conclusión, no obstante, es más válida para las dimensiones de extraversión y neuroticismo, las dos dimensiones, sin duda, más relevantes del cuestionario. Aunque de acuerdo con nuestros resultados la versión española del EPQR-A reúne las mínimas propiedades psicométricas para su utilización, las subescalas de psicoticismo y sinceridad deben usarse con la reservas propias que se han venido haciendo a ambas dimensiones del EPQ, especialmente a la subescala de psicoticismo. El EPQR-A, por tanto, puede ser una alternativa válida a los cuestionarios EPQ y EPQR, pruebas estas mucho más largas y a veces innecesariamente tediosas. 


\section{REFERENCIAS BIBLIOGRÁFICAS}

Caruso, J.C., Witkiewitz, K., Belcourt, A., y Gottlieb, J.D. (2001). Reliability of scores from the Eysenck Personality Questionnaire: A reliability generalization study. Educational and Psychological Measurement, 61, 675-689.

Eysenck, H.J., y Eysenck, S.B.G. (1983). EPQ: Cuestionario de personalidad. Madrid: Técnicos Especialistas Asociados.

Forrest, S., Lewis, C.A., y Shevlin, M. (2000). Examining the factor structure and differential functioning of the Eysenck Personality Questionnaire Revised-Abbreviated. Personality and Individual Differences, 29, 579-588.

Francis, L.J. (1993). The dual nature of the Eysenckian neuroticism scales: A question of sex differences. Personality and Individual Differences, 15, 43-59.

Francis, L.J., Brown, L.B., y Philipchalk, R. (1992). The development of an abbreviated form of the Revised Eysenck Personality Questionnaire (EPQR-A): Its use among students in England, Canada, the U.S.A and Australia. Personality and Individual Differences, 13, 443-449.

Joiner, Jr., T.E., Sandín, B., Chorot, P., Lostao, L., y Marquina, G. (1997). Development and factor-analytic validation of the SPANAS among women in Spain: (More) cross-cultural convergence in the structure of mood. Journal of Personality Assessment, 68, 600-615.

Katz, Y.J., y Francis, L.J. (2000). Hebrew revised Eysenck Personality Questionnaire: Short form (EPQR-S) and abbreviated form (EPQR-A). Social Behavior and Personality, 28, 555-560.

Maltby, J., y Talley, M. (1998). The psychometric properties of an abbreviated form of the Revised Junior Eysenck Personality Questionnaire (JEPQR-A) among 12-15year-old U.S. young persons. Personality and Individual Differences, 24, 891-893.

Meyer, T.J., Miller, M.L., Metzger, R.L., y Borkovec, T.D. (1990). Development and validation of the Penn State Worry Questionnaire. Behaviour Research and Therapy, 28, 487-495.
Peterson, R.A., y Reiss, R.J. (1992). Anxiety Sensitivity Index Manual (2nd edition). Worthington, $\mathrm{OH}$ : International Diagnostic Systems.

Sandín, B., y Chorot, P. (1991). Cuestionario de Preocupación PSWQ. Madrid: UNED (policopiado).

Sandín, B., Chorot, P., y McNally, R.J. (1996). Validation of the Spanish version of the Anxiety Sensitivity Index in a clinical sample. Behaviour Research and Therapy, 34, 283-209.

Sandín, B., Chorot, P., y McNally, R.J. (2001). Anxiety sensitivity index: Normative data and its differentiation from trait anxiety. Behaviour Research and Therapy, 39, 213219.

Sandín, B., Chorot, P., Lostao, L., Joiner, T.E., Santed, M.A., y Valiente, R.M. (1999). Escalas PANAS de afecto positivo y negativo: Validación factorial y convergencia transcultural. Psicothema, 11, 37-51.

Sandín, B., Chorot, P., Lostao, L., Valiente, R., Jiménez, P., y Santed (1995). Factor structure of the Spanish Penn State Worry Questionnaire. Póster. World Congress of Behavioural \& Cognitive Therapies. Copenhague (Dinamarca), 10-15 de julio.

Sandín, B., Valiente, R.M., y Chorot, P. (1999a). Evaluación del estrés psicosocial. En B. Sandín (Ed.), El estrés psicosocial: Conceptos y consecuencias clínicas (pp. 245-316). Madrid: Klinik.

Sandín, B., Valiente, R.M., y Chorot, P. (1999b). Material de apoyo para la evaluación de los miedos y fobias. En B. Sandín (Ed.), Las fobias específicas (pp. 161-188). Madrid: Klinik.

Sandín, B., Valiente, R.M., Chorot, P., Olmedo, M., y Santed, M.A. (2002). Versión española del EPQR-A (I): Análisis exploratorio de la estructura factorial. Revista de Psicopatología y Psicología Clínica, 7, 195-205.

Shevlin, M., Bailey, F., y Adamson, G. (2002). Examining the factor structure and sources of differential functioning of the Eysenck Personality Questionnaire Revised-Abbreviated. Personality and Individual Differences, 32, 479-487.

Spielberger, C.D., Gorsuch, R.R, y Lushene, R.E. (1982). STAI: Cuestionario de Ansie- 
dad Estado/Rasgo. Madrid: Técnicos Especialistas Asociados (TEA).

Tucker, L.R. (1951). A method for synthesis of factor analysis studies (Personnel Research

Section Report, No. 984; Contract DA49-083, Department of the Army). Princeton, NJ: Educational Testing Services.

Valiente, R., Sandín, B., Chorot, P., y Santed, M.A. (1996). Versión española del cuestionario EPQR-Abreviado: Datos preliminares con muestras españolas. Póster. XXXII
Congreso de la Sociedad Española de Medicina Psicosomática. Madrid, 21-23 de noviembre.

Watson, D., Clark, L.A., y Tellegen, A. (1988). Development and validation of brief measures of positive and negative affect: The PANAS scales. Journal of Personality and Social Psychology, 54, 1063-1070.

Wrigley, C.S., y Neuhaus, J.O. (1955). The matching of two sets of factors. American Psychologist, 10, 418. 